\title{
The Effect of the Number of Sets on Power Output for Different Loads
}

\author{
by \\ Antonio J. Morales-Artacho ${ }^{1}$, Paulino Padial ${ }^{1}$, Amador García-Ramos ${ }^{1}$, \\ Belén Feriche ${ }^{1}$
}

There is much debate concerning the optimal load $(\mathrm{OL})$ for power training. The purpose of this study was to investigate the effect of the number of sets performed for a given load on mean power output $\left(P_{\text {mean }}\right)$. Fourteen physically active men performed 3 sets of 3 bench-press repetitions with 30, 40 and $50 \mathrm{~kg}$. The highest mean power value ( $P_{\max }$ ) across all loads and $P_{\text {mean }}$ were compared when data were taken from the first set at each absolute load vs. from the best of three sets performed. Pmean increased from the first to the third set (from $5.99 \pm 0.81$ to $6.16 \pm 0.96 \mathrm{~W} \cdot \mathrm{kg}^{-1}, p=0.017$ ), resulting in a main effect of the set number $(p<0.05)$. At the $30 \mathrm{~kg}$ load $P_{\text {mean }}$ increased from the first to the third set (from $6.01 \pm 0.75$ to $\left.6.35 \pm 0.85 \mathrm{~W} \cdot \mathrm{kg}^{-1} ; p<0.01\right)$. No significant effect was observed at 40 and $50 \mathrm{~kg}$ loads $(p>0.05)$. $P_{\max }$ and velocity were significantly affected by the method employed to determine $P_{m e a n}$ at each load $(p<0.05)$. These results show a positive effect of the number of sets per load on $P_{m e a n}$, affecting $P_{\max }$, OL and potentially power training prescription.

Key words: power training, power test, strength, velocity, postactivation potentiation, bench press.

\section{Introduction}

The capacity to generate maximal upper body power has been suggested to be a determinant factor for success in sport activities requiring an optimal relationship between force and velocity. These may include throwing an object or explosively overcoming an external resistance (i.e. an opponent in judo or water in swimming) (Baker and Newton, 2005).

Maximal power is determined by optimal levels of force and velocity (Argus et al., 2013). Consequently, numerous studies have attempted to establish the load at which power is maximally developed (optimal load, OL) (Jandacka and Vaverka, 2008; Argus et al., 2013). Power-load and force-velocity curves are often based on a gradually increasing-load test while performing muscular tasks at maximal velocity (McMaster et al., 2014). Nevertheless, there is no general consensus in the literature regarding the exact procedure to obtain the force-velocity relationship during multijoint movements. Specifically, it is debatable how many cumulative exercise sets per load should be accomplished to accurately determine the OL, with numbers from 1 to 3 sets previously reported (Izquierdo et al., 2002; Lawton et al., 2006; Limonta and Sacchi, 2010; Marques et al., 2007; Sánchez-Medina et al., 2010; Thomas et al., 2007; Jandacka and Uchytil, 2011).

Earlier research has described the effect of preceding muscular activity on subsequent neuromuscular performance (Tillin and Bishop, 2009; Froyd et al., 2013; Wilson et al., 2013) as improvements in actions such as throwing, jumping, or hitting a mobile object (Gossen and Sale, 2000; Sale, 2002). Potential physiological mechanisms include increment in the number of

1 - Deparment of Physical Education and Sport. Faculty of Sport Sciences, University of Granada. 
actomyosin bridges, stimulation of the central nervous system (Rixon et al., 2007) and enhanced recruitment of motor units following prior activity (Güllich and Schmidtbleicher, 1996). Consequently, an upward and rightward shift in the force-velocity curve, induced by potentiation, has been previously proposed (Sale, 2002). It could potentially affect OL determination when performing multiple sets per load in order to obtain the force-velocity profile.

Among other factors, it seems that stimulus variability (Baudry and Duchateau, 2004; Gossen and Sale, 2000), training experience (Wilson et al., 2013), the muscle group examined (Pääsuke et al., 2007) or the time elapsed between the conditioning activity and the task (Gossen and Sale, 2000) may affect the balance between potentiation and fatigue. Previous research suggests that specificity of the conditioning activity is important when inducing post activation potentiation (Bonitch-Domínguez et al., 2010). Also, conditioning stimulus involving moderate intensities and including multiple sets has been proposed to be more efficient than heavy conditioning exercises (Wilson et al., 2013) in trained participants. All these factors could possibly affect power output and OL determination when performing various sets per load during the force-velocity curve protocol. It was thus hypothesised that power output performance and the OL could be significantly affected by the number of sets accomplished for a given load during the force-velocity curve.

Therefore, the purpose of the present study was to examine the effects of multiple sets of bench press repetitions conducted at submaximal progressive loads on the maximum power output and OL determination.

\section{Material and Methods}

\section{Participants}

Fourteen healthy, physically active male students were recruited from the student community of Granada University. All participants had a minimum of 3 years of experience in strength training. Age, body mass and height were $22.73 \pm 3.97$ years, $77.26 \pm 9.21 \mathrm{~kg}$ and $178.93 \pm 5.08 \mathrm{~cm}$, respectively. All participants read and signed an informed consent form. The study protocol adhered to the tenets of the Declaration of Helsinki and was approved by the
Ethics Committee of the Granada University.

\section{Measures}

An intra-group repeated measures design was employed to examine the influence of performing three sets versus only one set, for a given absolute load, on power output. A linear position transducer was used to measure concentric-phase barbell velocity during the bench press exercise. The absolute workloads tested were 30,40 and $50 \mathrm{~kg}$.

\section{Procedures}

In a single visit to the laboratory and following a standard warm-up, participants underwent a force-velocity profile assessment. It comprised 3 sets of 2-3 concentric-only benchpress repetitions with three increasing absolute loads (Figure 1). To avoid forward and backwards barbell displacements, testing was performed in a Smith machine (Technogym, Italy).

In general, the intensity linked to maximal power development is approximately $30 \%$ of the maximal isometric force (MIF) (Cormie et al., 2011). For the bench press exercise, the literature describes a range of $30-60 \% 1 \mathrm{RM}(35-70 \mathrm{~kg})$ as optimal for power development (McMaster et al., 2014; Castillo et al., 2011; Newton et al., 1997). Estimations of MIF for our participants (94.33 \pm $16.53 \mathrm{~kg}$ ) were made from the force-velocity curve. It provided evidence for the presence of the three absolute loads (30, 40 and $50 \mathrm{~kg}$ ) within the intensity range mentioned above (27 to $65 \%$ of the MIF).

Rest periods between sets were $3 \mathrm{~min}$ when barbell velocities were $\geq 1 \mathrm{~m} \cdot \mathrm{s}^{-1}$ and $5 \mathrm{~min}$ when the barbell velocity was slower than $1 \mathrm{~m} \cdot \mathrm{s}^{-1}$. Participants were instructed and verbally encouraged to achieve maximal velocity of the bar during each repetition. To ensure exclusive concentric-phase work, the barbell was kept still for $2 \mathrm{~s}$ pauses in the starting position (velocity $=0$ ) 3-5 $\mathrm{cm}$ above the chest.

Mechanical variables were obtained using a Real Power Pro electronic linear transducer (Globus Italia connected to a Tesys 400 system) and the ErgoSystem 8.5 software. The system was fixed to the bar using a clip such that the cable would be vertically displaced and track the position of the bar during its movement (1000 $\mathrm{Hz}$.

For each repetition, applied force $(F)$, velocity $(V)$ and power values were obtained. 
Data were expressed relatively to body weight $\left(\mathrm{W} \cdot \mathrm{kg}^{-1}\right)$. A single repetition was selected as the best for each set and load, corresponding to the highest mean power and designated as the mean power $\left(\mathrm{P}_{\text {mean }}\right)$. Maximal power $\left(\mathrm{P}_{\max }\right)$ was taken as the highest mean power recorded throughout the entire test. Then, $P_{\text {mean }}$ for each load and $P_{\max }$ of the test were obtained to compare the first set to the best of the 3 sets accomplished per each absolute load. The optimal load (OL) was defined as the mechanical load $(\mathrm{kg})$ at which $P_{\max }$ was achieved.

\section{Statistical Analysis}

Data are expressed as mean \pm standard deviation. Frequency distributions were assessed using the Shapiro-Wilk test. A two-way repeated measures ANOVA was employed to assess the influence of the set number ( $1^{\text {st }} \mathrm{vs} .2^{\text {nd }}$ vs. $\left.3^{\text {rd }}\right)$ and load (30 vs. 40 vs. $50 \mathrm{~kg}$ ) on power output. When an interaction or main effect occurred, pairwise comparisons were made using Bonferroni post hoc analysis. Greenhouse-Geisser correction was selected when the Mauchly's test of Sphericity was significant. The magnitude of the differences between both conditions was expressed as a standardized mean difference (Cohen's d effect size; ES). The criteria to interpret the magnitude of the ES were as follows: $<0.2=$ trivial, $0.2-0.6=$ small, $0.6-1.2=$ moderate, $1.2-2.0=$ large, $2-4.0=$ very large and $>4=$ extra-large (Hopkins et al., 2009).

Paired t-tests were used to compare $P_{\text {mean }}$ values achieved in the first set vs. the best of the 3 sets for each absolute load. Furthermore, paired ttests were also used to compare the force and velocity values linked to each $P_{\text {mean }}$ value selected.
Significance was set at $p \leq 0.05$ and all statistical analyses were performed in SPSS (v. 19).

\section{Results}

There was a main effect of the set number (ANOVA, $p=0.028 ; F_{\exp }=4.10 ; 2,26 d f$ ) due to an increase in $P_{\text {mean }}$ from the first to the third set (5.99 \pm 0.81 vs. $6.16 \pm 0.96, p=0.017$, respectively). Pairwise comparisons revealed that these differences were due to an increase in $P_{\text {mean }}$ from the first to the third set at the $30 \mathrm{~kg}$ load $(6.01 \pm$ 0.75 to $6.35 \pm 0.85 \mathrm{~W} \cdot \mathrm{kg}^{-1}$, respectively; $p<0.01$ ). For the remaining workloads, no significant effects of the set number on $P_{\text {mean }}$ were observed $(p>0.05)$.

There was a main effect of the load (ANOVA, $p=0.003 ; F_{\text {exp }}=10.39 ; 1.3,17.1 d f$ ) taking into account lower $P_{\text {mean }}$ values with $50 \mathrm{~kg}$ compared to the $30 \mathrm{~kg}(p=0.012)$ and $40 \mathrm{~kg}$ loads $(p=0.001)$. No differences were observed in Pmean values attained with 30 and $40 \mathrm{~kg}(p=0.646)$. No set number by load interaction effect was shown $(p=0.249)$. Inter set and inter load comparisons are shown in Table 1.

At each load, significant differences were observed in Pmean when the first set vs. the best of the three sets was compared (t-test, $p<0.01$; Table 2). $P_{\text {mean }}$ was located in the $2.20 \pm 0.77$ set number for $30 \mathrm{~kg}$, the $2.33 \pm 0.72$ set number for $40 \mathrm{~kg}$ and the $2.00 \pm 0.78$ set number for $50 \mathrm{~kg}$.

$P_{\max }$ and mean velocity were also significantly affected by the method employed to determine Pmean for each load $(p<0.05$; Table 3). Applied force and the OL linked to $P_{\text {mean }}$ values are also shown in Table 3.

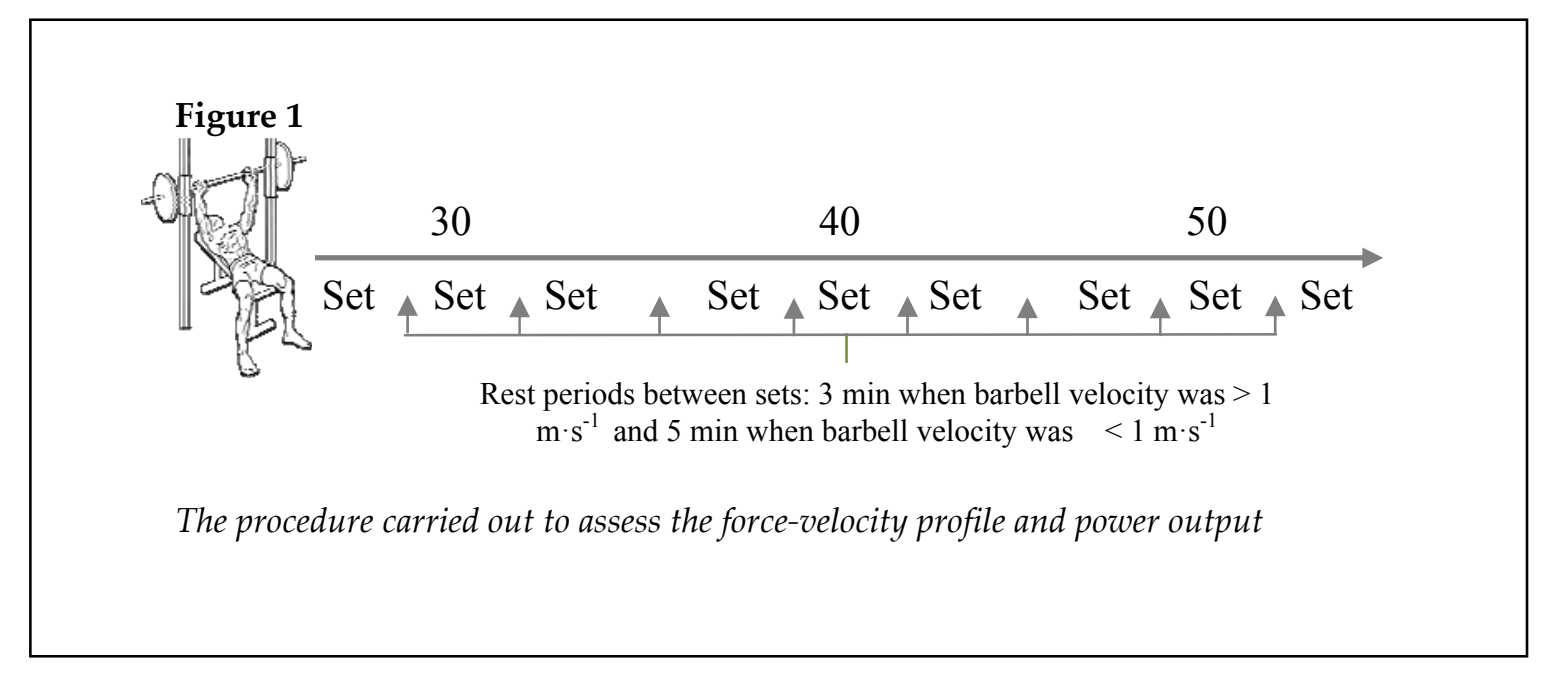




\section{Table 1}

Mean power outputs relative to body mass recorded for increasing numbers of bench press repetitions (intra-load) performed at increasing training loads (inter-load)

\begin{tabular}{|c|c|c|c|c|}
\hline $\begin{array}{l}P_{\text {mean }} \\
\mathrm{W} \cdot \mathrm{kg}^{-1}\end{array}$ & $30 \mathrm{~kg}$ & $40 \mathrm{~kg}$ & $50 \mathrm{~kg}$ & $P_{2}$ \\
\hline $1^{\text {st }}$ set & $6.01 \pm 0.75$ & $6.21 \pm 0.86$ & $5.74 \pm 0.92^{b}$ & 0.002 \\
\hline $2^{\text {nd }}$ set & $6.27 \pm 0.78$ & $6.22 \pm 0.93$ & $5.81 \pm 1.03^{c}$ & 0.008 \\
\hline $3^{\text {rd }}$ set & $6.35 \pm 0.85^{*}$ & $6.31 \pm 0.99$ & $5.82 \pm 1.17 b, c$ & 0.005 \\
\hline$P_{1}$ & 0.005 & 0.505 & 0.666 & \\
\hline
\end{tabular}

Data expressed as observed mean values $\pm S D$. $P_{\text {mean }}=$ mean power; $P_{1}=$ intra-load comparisons ( $\left.A N O V A\right) ; P_{2}=$ inter-load comparisons (ANOVA). $\boldsymbol{a}$ = difference 30 versus $40 \mathrm{~kg} ; \boldsymbol{b}=$ difference 40 versus $50 \mathrm{~kg}$; $c=$ difference 30 versus $50 \mathrm{~kg}$; *difference set 1 versus $3(p=0.017)$.

Table 2

Mean power output from the first and best set from each absolute load

\begin{tabular}{|c|c|c|c|c|}
\hline & & $30 \mathrm{~kg}$ & $40 \mathrm{~kg}$ & $50 \mathrm{~kg}$ \\
\hline $1^{\text {st }}$ Set $\left(W \cdot \mathrm{kg}^{-1}\right)$ & & $6.01 \pm 0.75$ & $6.21 \pm 0.86$ & $5.74 \pm 0.92$ \\
\hline Best Set $\left(\mathrm{W} \cdot \mathrm{kg}^{-1}\right)$ & & $6.46 \pm 0.77$ & $6.48 \pm 0.90$ & $5.97 \pm 1.06$ \\
\hline$p$ & & $<0.001$ & $<0.001$ & 0.013 \\
\hline \multirow[b]{2}{*}{$95 \% \mathrm{CI}$} & LL & -0.265 & -0.171 & -0.055 \\
\hline & UP & -0.630 & -0.384 & -0.394 \\
\hline ES & & 0.59 & 0.31 & 0.23 \\
\hline
\end{tabular}

Data expressed as observed mean power values \pm SD. $1^{\text {st }}$ Set $=$ highest mean power value observed at the first set of each load; Best Set = highest mean power value achieved in all sets performed at each absolute loads; $C I=$ confidence interval; lower limit (LL); upper limit (UP); $p=$ probability error ES = effect size. 


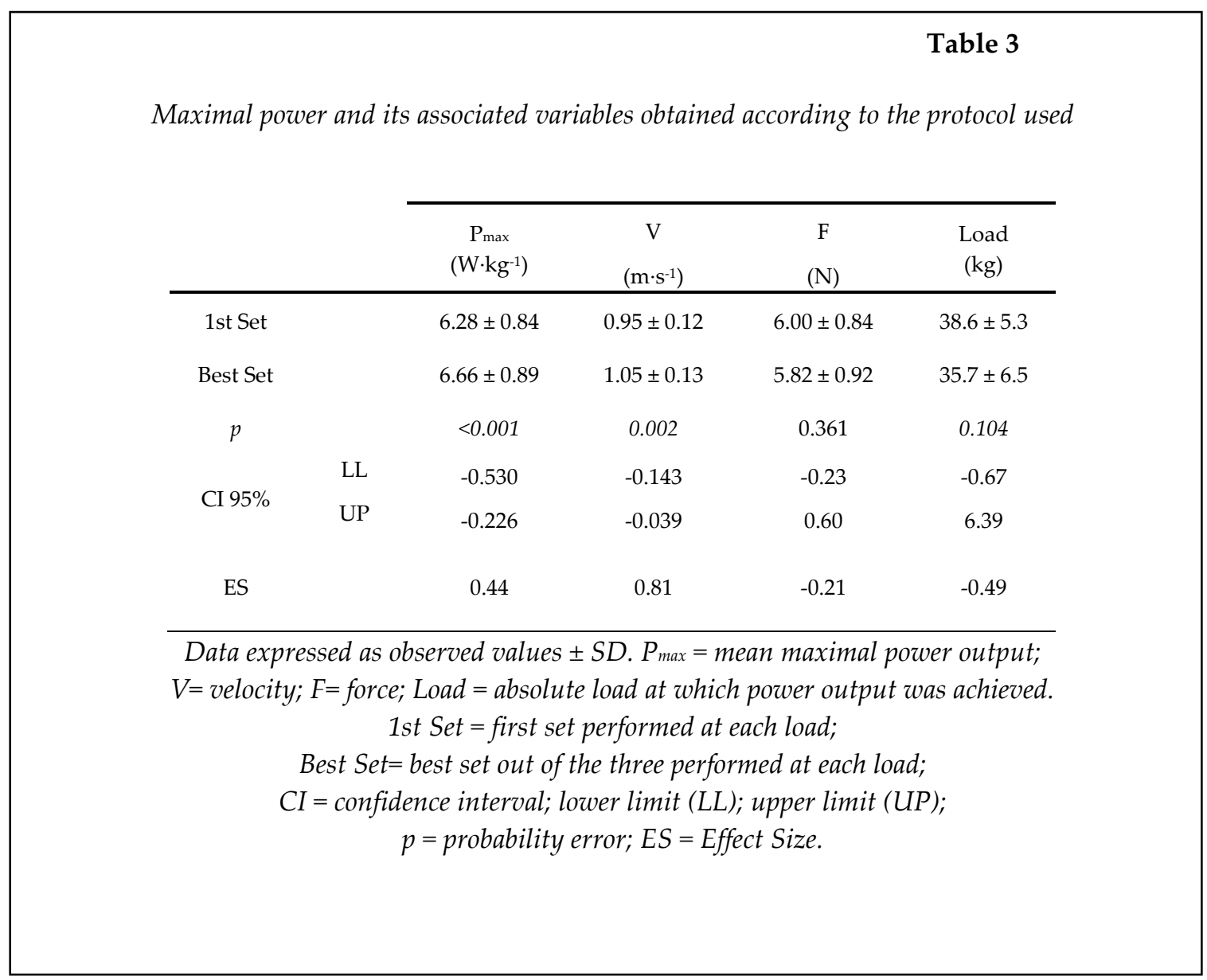

\section{Discussion}

The main finding of the present study is that the number of sets conducted at a given load affects maximal power output and OL determination. Consequently, the three cumulative bench press sets influenced positively power output. Our results may be of interest for sports practitioners and scientists when testing and prescribing power strength training.

Post activation potentiation effects have previously been reported in vitro (Metger et al., 1989) and in vivo conditions (Sale, 2002). Training studies have shown acute improvements in power and explosive strength performance following protocols with moderate and heavy loads (Güllich and Schmidtbleicher, 1996; Young et al., 1998; Ferreira et al., 2012). However, to the best of our knowledge, this is the first study to directly address the practicality of post activation potentiation in the OL determination and power development testing. Our results showed a $P_{\max }$ improvement and a tendency for OL decrement when the highest power output was selected from the three sets per load instead of using only the first set.

The effects of post activation may be confounded by factors such as variations in the type of stimulus (Baudry and Duchateau, 2004; Gossen and Sale, 2000), the muscle group assessed (Pääsuke et al., 2007) or training experience of participants (Wilson et al., 2013). In line with previous studies (Bonitch-Domínguez et al., 2010), our results suggest that potentiation effects can be observed when using the same exercise and load as conditioning activity.

In addition, the rest period between conditioning activity and performance assessment is known to be important to allow optimal balance between fatigue and potentiation effects (Gossen and Sale, 2000). Previous investigations have attributed the lack of potentiation effects to insufficient rest periods for a given volume of conditioning work (Gossen and Sale, 2000; Chaouachi et al., 2011). Based on the velocity of barbell displacement, we adjusted rest periods so 
that participants rested longer (5 $\mathrm{min}$ ) between sets when the capacity to develop force quickly (barbell velocity) decreased. However, and despite of the low volume of work performed (2 3 repetitions per set), no effect of the set number was observed at 40 and $50 \mathrm{~kg}$ loads, presumably due to fatigue accumulation (Table 1). Previous research has reported optimal potentiation effects in bench-press $7 \mathrm{~min}$ after 1RM (Ferreira et al., 2012), which might suggest that our rest periods were not long enough at higher workloads. Notwithstanding, significant $P_{\text {mean }}$ increments were observed when all sets at each load were taken into account (Table 2).

Training experience is also known to be an important factor that may affect balance between a rest period and volume of work (Wilson et al., 2013; Tillin and Bishop, 2009). Our participants were moderately trained, however, no fatigue symptoms were reflected due to similar power output from the 1st to $3 \mathrm{rd}$ set at the $50 \mathrm{~kg}$ load. Hence, it seems that interactions among all these factors may determine the relationship between optimal volume, workload and recovery to induce an optimal potentiating effect.

In agreement with Sale's (2002) hypothesis, our results showed an effect of the set number on Pmean, inducing an upward and leftward shift in the power-load curve. This is supported by previous research which linked the efficiency of prior explosive exercise to subsequent increments in electrical muscle activity (Bigland-Ritchie et al., 1986; Gossen and Sale, 2000), likely due to higher calcium sensitivity (Sale, 2002). This observation establishes a need to define the ideal protocol to efficiently measure $P_{\max }$ and OL. These results add information to the existing void concerning power-load curve protocols, which have usually included one (Thomas et al., 2007) or two (Jandacka and
Vaverka, 2008; Izquierdo et al., 2002) sets per load. When the best set vs. the first was selected, $P_{\max }$ was improved by $\sim 6 \%$, and barbell velocity increased by $\sim 10 \%$.

A few limitations of the present investigation should be taken into account when interpreting our results. The fact that testing procedures involved concentric-only bench press exercise (non-ballistic) may be responsible for underestimations of power performance. Cronin et al. (2003) reported greater velocity development, but not strength, when the bar was released compared with concentric-only bench press exercise. Notwithstanding, Frost et al. (2008) showed that when the lifting phase was limited to only positive work, the difference between ballistic (i.e. bench press thrown) and non-ballistic bench press exercises was not significant in loads above 30\% 1RM. Nevertheless, although similar results would be expected during ballistic benchpress exercise, further research involving upper and lower body exercises is needed to firmly understand potentiation phenomenon in muscle power assessments.

In conclusion, our findings indicate an effect of the number of sets conducted at each load on power and velocity measurements. If only one set per load was performed, the training load linked to maximal power would be overestimated, affecting the barbell velocity as well. When we only considered the first set of bench press repetitions, $P_{\max }$ was underestimated and the associated load was overestimated by $\sim 8 \%(\mathrm{p}<0.05)$. By including two sets per load in the assessment protocol, we can avoid overestimating the OL and maximal power. This could have a significant impact on power training prescription and training induced adaptations.

\section{References}

Argus C, Nicholas D, Keogh J, Hopkins W. Assessing the variation in the load that produces maximal upperbody power. The Journal of Strength \& Conditioning Research, 2013; 28: 240-244

Baker D, Newton RU. Methods to Increase the Effectiveness of Maximal Power Training for the Upper Body. Strength Cond J, 2005; 27: 24-32

Baudry S, Duchateau J. Postactivation potentiation in human muscle is not related to the type of maximal conditioning contraction. Muscle Nerve, 2004; 30: 328-336

Bigland-Ritchie B, Furbush F, Woods JJ. Fatigue of intermittent submaximal voluntary contractions: central 
and peripheral factors. J Appl Physiol, 1986; 61: 421-429

Bonitch-Dominguez J, Bonitch-Gongora J, Padial P, Feriche B. Changes in peak leg power induced by successive judo bouts and their relationship to lactate production. J Sports Sci, 2010; 28: 1527-1534

Castillo F, Valverde T, Morales A, Pérez-Guerra A, de León F, García-Manso JM. Maximum power, optimal load and optimal power spectrum for power training in upper-body (bench press): a review. Rev Andal Med Deporte, 2011; 5: 18-27

Chaouachi A, Poulos N, Abed F, Turki O, Brughelli M, Chamari K, Drinkwater EJ, Behm DG. Volume, intensity, and timing of muscle power potentiation are variable. Appl Physiol Nutr Metab, 2011; 36: 736747

Cormie P, McGuigan MR, Newton RU. Developing maximal neuromuscular power: Part 1--biological basis of maximal power production. Sports Med, 2011; 41: 17-38

Cronin JB, McNair PJ, Marshall RN. Force-velocity analysis of strength-training techniques and load: implications for training strategy and research. J Strength Cond Res, 2003; 17: 148-155

Ferreira SL, Panissa VL, Miarka B, Franchini E. Post activation potentiation: effect of various recovery intervals on bench press power performance. J Strength Cond Res, 2012; 26: 739-744

Froyd C, Beltrami F, Jensen J, Noakes T. Potentiation Increases Peak Twitch Torque by Enhancing Rates of Torque Development and Relaxation. J Hum Kinet, 2013; 38: 83-94

Gossen ER, Sale DG. Effect of postactivation potentiation on dynamic knee extension performance. Eur J Appl Physiol, 2000; 83: 524-530

Güllich A, Schmidtbleicher D. MVC-induced short-term potentiation of explosive force. New Stud Athlet, 1996; 11: 67-81

Hopkins WG, Marshall SW, Batterham AM, Hanin J. Progressive statistics for studies in sports medicine and exercise science. Med Sci Sports Exerc, 2009; 41: 3-13

Izquierdo M, Hakkinen K, Gonzalez-Badillo JJ, Ibanez J, Gorostiaga EM. Effects of long-term training specificity on maximal strength and power of the upper and lower extremities in athletes from different sports. Eur J Appl Physiol, 2002; 87: 264-271

Jandacka D, Uchytil J. Optimal load maximizes the mean mechanical power output during upper extremity exercise in highly trained soccer players. J Strength Cond Res, 2011; 25: 2764-2772

Jandacka D, Vaverka F. A regression model to determine load for maximum power output. Sports Biomech, 2008; 7: 361-371

Lawton TW, Cronin JB, Lindsell RP. Effect of interrepetition rest intervals on weight training repetition power output. J Strength Cond Res, 2006; 20: 172-176

Limonta E, Sacchi M. Morphological analysis of force/velocity relationship in dynamic exercise at varying loads. J Strength Cond Res, 2010; 24: 2065-2072

Marques MC, van den Tilaar R, Vescovi JD, Gonzalez-Badillo JJ. Relationship between throwing velocity, muscle power, and bar velocity during bench press in elite handball players. Int J Sports Physiol Perform, 2007; 2: 414-422

McMaster D, Gill N, Cronin J, McGuigan M. A brief review of strength and ballistic assessment methodologies in sport. Sports Med, 2014; 44: 603-623

Metzger JM, Greaser ML, Moss RL Variations in cross-bridge attachment rate and tension with phosphorylation of myosin in mammalian skinned skeletal muscle fibers. Implications for twitch potentiation in intact muscle. J Gen Physiol, 1989; 93: 855-883

Newton RU, Murphy AJ, Humphries BJ, Wilson GJ, Kraemer WJ, Hakkinen K. Influence of load and stretch 
shortening cycle on the kinematics, kinetics and muscle activation that occurs during explosive upperbody movements. Eur J Appl Physiol Occup Physiol, 1997; 75: 333-342

Pääsuke M, Saapar L, Ereline J, Gapeyeva H, Requena B, Oopik V. Postactivation potentiation of knee extensor muscles in power- and endurance-trained, and untrained women. Eur J Appl Physiol, 2007; 101: $577-585$

Rixon KP, Lamont HS, Bemben MG. Influence of type of muscle contraction, gender, and lifting experience on postactivation potentiation performance. J Strength Cond Res, 2007; 21: 500-505

Sale DG. Postactivation potentiation: role in human performance. Exerc Sport Sci Rev, 2002; 30: 138-143

Sánchez-Medina L, Perez CE, Gonzalez-Badillo JJ. Importance of the propulsive phase in strength assessment. Int J Sports Med, 2010; 31: 123-129

Thomas GA, Kraemer WJ, Spiering BA, Volek JS, Anderson JM, Maresh CM. Maximal power at different percentages of one repetition maximum: influence of resistance and gender. J Strength Cond Res, 2007; 21: $336-342$

Tillin NA, Bishop D. Factors modulating post-activation potentiation and its effect on performance of subsequent explosive activities. Sports Med, 2009; 39: 147-166

Wilson J, Duncan N, Marin P, Brown L, Loenneke J, Wilson S, Jo E, Lowery R, Ugrinowitsch C. Metaanalysis of post activation potentiation and power: effects of conditioning activity, volume, gender, rest periods, and training status. J Strength and Cond Res, 2013; 27: 854-859

Young WB, Jenner A, Griffiths K. Acute Enhancement of Power Performance From Heavy Load Squats. J Strength and Cond Res, 1998; 12: 82-84

\section{Corresponding author:}

\section{Belén Feriche Castanys}

Department of Physical Education and Sport. Faculty of Sport Sciences

University of Granada, Ctra. Alfacar, s/n, 18011, Granada (Spain)

E-mail: mbelen@ugr.es 